\title{
Principios Biomecánicos en Inestabilidad Crónica Posterolateral de Rodilla
}

\section{Biomechanical Principles of Chronic Posterolateral Knee Instability}

\author{
Pedro Alejandro Díaz Allende ${ }^{1}$ \\ ${ }^{1}$ Traumatólogo, Servicio Ortopedia y Traumatología Hospital \\ Ricardo Valenzuela Sáez, Rengo, Chile \\ Address for correspondence Pedro Alejandro Díaz Allende, MD, \\ Traumatólogo, Servicio Ortopedia y Traumatología Hospital Ricardo \\ Rev Chil Ortop Traumatol 2017;58:41-47. \\ Valenzuela Sáez, Rengo, Chile (e-mail: drpedrodiaz@gmail.com).
}
Resumen
Palabras Clave
- anatomía
- biomecánica
- esquina posterolateral
- evaluación clínica
- inestabilidad crónica
- rodilla
Abstract
Keywords
- anatomy
- biomechanics
- chronic instability
- clinical evaluation
- knee
- posterolateral corner

La inestabilidad crónica posterolateral de rodilla continúa siendo un tema desafiante desde la evaluación clínica hasta la resolución definitiva. La comprensión de la anatomía, biomecánica articular y las adaptaciones evolutivas de la rodilla para su complejo funcionamiento, son esenciales para el razonamiento diagnóstico y terapéutico de esas lesiones.
Chronic posterolateral knee instability continues to be a challenging issue from clinical evaluation to final resolution. The understanding of anatomy, joint biomechanics and evolutionary adaptations of the knee for its complex functioning are essential for the diagnostic and therapeutic reasoning of these injuries.

\section{Introducción}

Las lesiones crónicas de la esquina posterolateral (EPL) de rodilla, son infrecuentes y generalmente subdiagnosticadas. La historia clínica habitual en estos pacientes, no reporta un antecedente traumático específico y la insuficiencia por elongación ligamentaria se debe usualmente a trastornos del alineamiento óseo subyacentes. Su alta asociación con lesiones del ligamento cruzado posterior $(60 \%){ }^{1}$ van alterando la biomecánica articular de forma secuencial produciendo inestabilidad poliaxial, lo que genera un patrón de marcha característico conocido como varus thrust.

En ese tipo de lesiones, es obligatorio un examen físico exhaustivo que implique una evaluación de la marcha, alineamiento de extremidades en bipedestación y test clínicos específicos.

La comprensión de la anatomía y biomecánica de la insuficiencia crónica de la EPL, permiten entender el razonamiento diagnóstico y terapéutico de esas lesiones. received

January 11, 2017

accepted

July 18, 2017

published online

August 23, 2017
DOI https://doi.org/

10.1055/s-0037-1606126. ISSN 0716-4548.
Copyright (e) 2017 by Thieme Revinter

Publicações Ltda, Rio de Janeiro, Brazil
License terms

(ㄷ) (i) $\ominus$ (\$) 


\section{Reseña Anatómica}

La estabilidad posterolateral de la rodilla es otorgada por numerosos componentes estáticos y dinámicos. ${ }^{2}$ Dentro de los primeros, se incluyen los ligamentos: colateral (fibular) lateral (LCL), fabelofibular, poplíteofibular (LPF), poplíteo oblicuo (LPO), tibiofibulares proximales (anterior y posterior) y el engrosamiento capsular lateral que comprende los ligamentos meniscofemorales y meniscotibiales (coronarios), del menisco externo. Dentro de los componentes dinámicos, destacan el músculo bíceps femoral (cabeza larga y corta), banda iliotibial, cabeza lateral del gastrocnemio y el tendón poplíteo (TP).

Gollehon y $\operatorname{col}^{3}$ describió a través de una técnica de resección anatómica selectiva, que las principales estructuras que contribuyen a la estabilidad en varo, rotación externa y traslación tibial posterolateral son el LCL, LPF y el TP. De tal forma que son esas 3 estructuras las que definen los componentes esenciales de la EPL. Los trabajos conducidos posteriormente por el Dr. Robert LaPrade en la materia, ${ }^{4}$ han permitido un acabado conocimiento de las relaciones anatómicas cualitativas y cuantitativas, orientadas a racionalizar el manejo de lesiones agudas ${ }^{5}$ y crónicas.

El LCL mide $71 \mathrm{~mm}$ de largo. Se origina $1.4 \mathrm{~mm}$ proximal y $3.1 \mathrm{~mm}$ posterior al epicóndilo lateral del fémur en una pequeña depresión ósea, insertándose en la porción anterolateral de la cabeza del peroné. Es el estabilizador primario en respuesta al estrés en varo y secundario a la rotación externa en los primeros grados de flexión. Tiene una fuerza máxima de $295 \mathrm{~N}^{6}$ y su mejor resistencia se observa entre $0^{\circ}-30^{\circ}$ de flexión de la rodilla. ${ }^{7}$

El TP mide 55mm de largo y funciona en conjunto con el LPF como restrictor primario de la rotación tibial externa. Se origina en el fémur a una distancia $18,5 \mathrm{~mm}$ más distal y anterior que el LCL (esa relación es clave como referencia en las reconstrucciones anatómicas de la EPL). Desde su origen sigue un trayecto oblicuo y profundo al LCL, en dirección posteroinferior, ingresando a través de la capsula articular al hiato poplíteo, de $13 \mathrm{~mm}$ de largo. Allí se ancla al menisco externo a través de 3 fascículos (anteroinferior, posterosuperior y posteroinferior). En el aspecto posteromedial de la cabeza del peroné se genera la unión músculo tendínea. Como músculo poplíteo, se inserta de forma amplia en la región posterior y proximal de la tibia. Presenta acciones secundarias en la estabilidad rotacional interna, varo y traslación tibial anterior. Su fuerza máxima es de $700 \mathrm{~N}^{6}$ y su mejor resistencia se observa en $60^{\circ}$ de flexión de la rodilla. ${ }^{7}$

El LPF (previamente conocido como ligamento arcuato) mide $15 \mathrm{~mm}$ de largo. Cursa desde la unión musculotendínea del poplíteo en dirección distal y lateral, uniéndose al aspecto posteromedial de la estiloides fibular en un ángulo de $38^{\circ}$ desde la vertical. Es un estabilizador primario en respuesta a la rotación tibial externa y secundario sobre la traslación tibial posterior e hiperextensión. Su fuerza máxima es de $298 \mathrm{~N}^{6}$ y su mejor resistencia se observa en $60^{\circ}$ de flexión de rodilla. $^{7}$ Aunque la función del LCL y TP han sido históricamente apreciados como esenciales en la estabilización lateral de la rodilla, el LPF ha recibido comparativamente menor atención. Sin embargo, esa estructura juega un rol preponderante. McCarthy et al. ${ }^{8}$ determinaron que la manera de lograr preservar la cinemática normal de la rodilla (ante una lesión aguda de la $\mathrm{EPL}$ ), debe incluir la reconstrucción de ese ligamento.

El LPO es la estructura ligamentaria más grande del aspecto posterior de la rodilla. ${ }^{9}$ En promedio, mide $12 \mathrm{~mm}$ de ancho por $48 \mathrm{~mm}$ de largo. Medialmente se origina como confluencia de la expansión capsular del ligamento oblicuo posterior y de la expansión lateral del tendón semimembranoso. Continúa lateralmente como una banda fascial ancha atravesando la región posterior (capsular) de la rodilla, presentando inserciones en el aspecto posterolateral de ésta, a nivel de la fabela (sesamoideo dentro de la porción proximal del tendón gastrocnemio lateral, en estado óseo solo en el 30\% de los casos), porción meniscofemoral de la capsula articular y músculo plantar. Por lo tanto, en su inserción presenta puntos de anclaje en común con el ligamento fabelofibular y la prolongación capsular de la cabeza corta del bíceps femoral. Su importante función es ser el estabilizador primario ante hiperextensión de rodilla, restringiendo el recurvatum. ${ }^{10}$

El nervio peroneo común emerge de la bifurcación del nervio ciático en el aspecto posterior del muslo y está formado por ramos de las raíces nerviosas espinales L4-S2. Cursa a lo largo del bíceps femoral y alrededor del cuello de la fíbula, separándose compartimentalmente a nivel de la pierna en los nervios peroneo superficial (compartimiento lateral) y profundo (compartimiento anterior). Su función motora incluye la extensión del hallux, la eversión y dorsiflexión del pie. Su función sensitiva se encuentra a nivel del tercio lateral y distal de la pierna, dorso del pie y primer espacio interdigital. Se estima que el $15 \%$ de los pacientes con lesión crónica de la EPL pueden desarrollar trastornos sensitivo-motores locales producto de su lesión (pie en gota). ${ }^{11}$

El ligamento cruzado posterior (LCP) con sus bandas anterolateral y posteromedial, actúan de forma sinérgica y codominante en restringir de forma primaria la traslación tibial posterior y secundariamente la hiperextensión, varo y rotación tibial externa, particularmente entre los $90^{\circ}-120^{\circ}$ de flexión. ${ }^{12}$ La completa insuficiencia del LCP resulta en un aumento de $11,4 \mathrm{~mm}$ en la traslación tibial posterior en $90^{\circ}$ de flexión, sin embargo la sección aislada de alguna de sus bandas no genera un incremento clínicamente significativo de esa traslación, ${ }^{13}$ siendo validado el principio de interdependencia de las bandas. ${ }^{14}$

\section{Biomecánica de la Inestabilidad Crónica Posterolateral}

En condiciones normales, la rodilla se encuentra sometida a cargas cíclicas impuestas por la marcha. Durante la fase de apoyo, existen 2 peaks de carga que equivalen a 3 veces el peso corporal. ${ }^{15}$ El primero, es generado por el descenso del talón cuando apoya el suelo (fase de apoyo inicial) y el segundo, cuando éste se eleva y el antepie se prepara para impulsar la masa corporal hacia adelante (fase de apoyo final).

Existen consideraciones importantes respecto al balance de fuerzas que pasan a través de la rodilla en el plano coronal. Durante la marcha en condiciones normales, el pie de apoyo 
permanece en línea con el centro de gravedad y la fuerza de reacción al peso del cuerpo tiene un vector que pasa medial al ancho de la rodilla, generando el momento aductor.

Por lo tanto, los 2 peaks de carga en fase de apoyo actúan en el compartimiento medial de la rodilla cuando la extremidad inferior se encuentra en aducción. En condiciones fisiológicas, el momento aductor es suficiente para generar una descarga temporal del compartimiento femorotibial (FT) externo, con la fuerza de compresión ejerciéndose por completo a través del compartimiento FT medial. ${ }^{16}$ La estabilidad en el plano coronal en esas circunstancias, dependerá de la tensión transitoria ejercida principalmente sobre la banda iliotibial, el ligamento colateral lateral y los abductores de cadera.

La geometría articular de la rodilla está adaptada a ese patrón de cargas, siendo más congruente el compartimiento FT medial, donde el cóndilo femoral descansa sobre un platillo cóncavo, de manera tal que el área de contacto articular es amplio, disminuyendo el estrés de compresión en comparación con el compartimiento FT lateral, que posee un platillo tibial convexo. En condiciones patológicas de varo articular (-Fig. 1), la consecuencia de esos peaks de carga en el compartimiento FT medial, generan cambios en el alineamiento y mayor aducción, lo que conduce a un círculo vicioso de incremento de carga medial y destrucción articular. $^{17,18}$

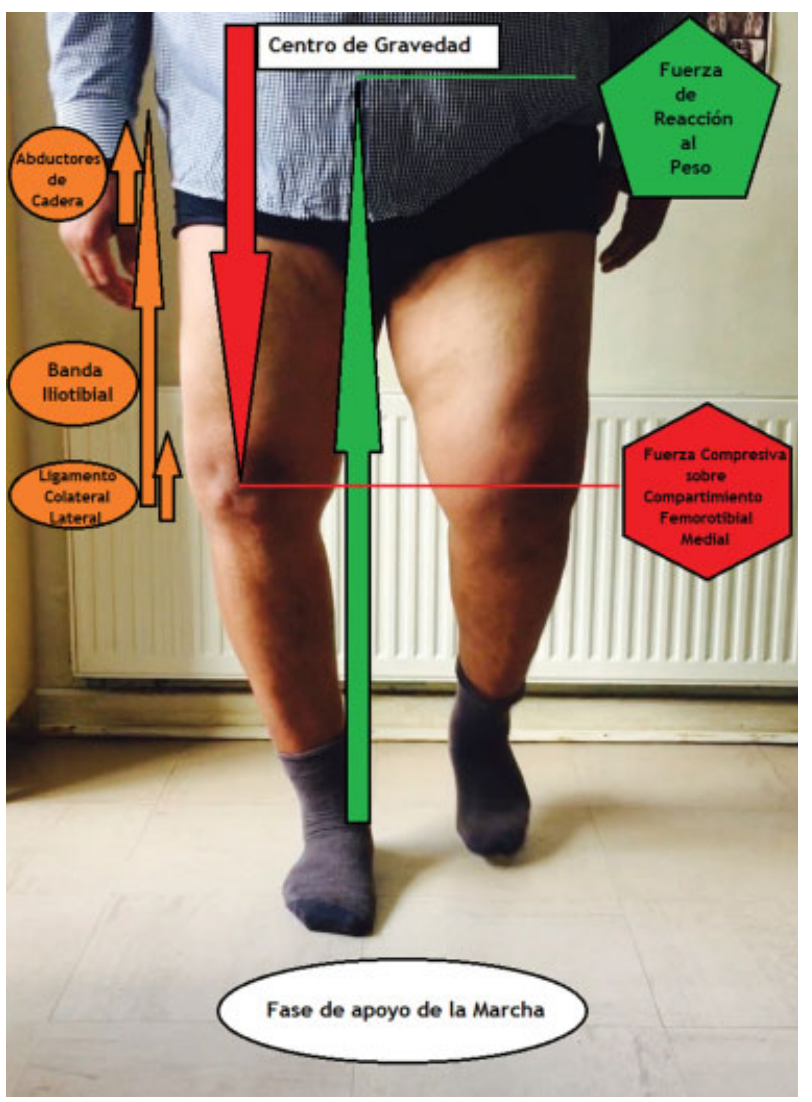

Fig. 1 Biomecánica del paciente con lesión crónica de la esquina posterolateral (EPL). Momento Aductor aumentado y varus thrust durante la marcha.
El reciente concepto de varo constitucional descrito por Bellemars y $\mathrm{col}^{19}{ }^{19}$ aporta al entendimiento biomecánico de ese punto. El estudio reporta que en una población adulta sana, existe un $32 \%$ de hombres y $17 \%$ de mujeres con un varo mecánico fisiológico de 3 o más grados, que postula se debe a las consecuencias de la Ley de Hueter-Volkmann. Ésta asevera que durante la infancia, el crecimiento en las fisis es inversamente proporcional a las cargas compresivas locales ejercidas sobre ella. Los incrementos cíclicos de carga en el compartimiento femorotibial medial, causados por el momento aductor en la rodilla durante la deambulación y la actividad física de impacto, podrían conducir al desarrollo de un alineamiento en varo, secundario al crecimiento retrasado en el compartimiento FT medial y acelerado en la fisis del compartimiento FT lateral. Vandekerckhove y col, ${ }^{20}$ concluye en base a ese concepto, que el varo constitucional contribuye significativamente al desarrollo de la gonartrosis medial.

Esas observaciones proveen una racionalidad biomecánica a la osteotomía valguizante, la cual desplaza lateralmente las fuerzas de reacción al peso durante la marcha, reduciendo el momento aductor y el estrés sobre el compartimiento FT medial.

Específicamente en el paciente con insuficiencia posterolateral, existe un aumento del momento aductor, que resulta en un aumento cíclico de la carga en el compartimiento femorotibial medial, estrechándolo. ${ }^{21}$ A su vez, las estructuras de sostén lateral que actúan como banda de tensión para contrarrestar la apertura articular externa durante la fase de apoyo monopodal, se comienzan a elongar progresivamente, produciendo un círculo vicioso de progresión de la inestabilidad (-Fig. 1). Todos los efectos anteriormente mencionados, se hacen más evidentes ante la insuficiencia concurrente del LCP.

Una lesión crónica aislada del LCP causa una traslación posterior de la tibia asociado a una rotación externa sobre el fémur. La cascada de alteraciones que genera la lesión del LCP se traducen en un aumento de alrededor de $30 \%$ en la presión del compartimiento medial de la rodilla. ${ }^{22}$

También existen incrementos de presión en la articulación patelofemoral a expensas, principalmente, de la faceta lateral (debido a la rotación femoral interna relativa a la consecuencia de la rotación tibial externa), y al polo inferior de la rótula (debido a la traslación tibial posterior que incrementa la tensión en el tendón patelar en alrededor de un 16\%). ${ }^{22}$ Como es de esperar, las lesiones meniscales asociadas aumentan de un $22 \%$ a un $36 \%$ de los casos si comparamos la fase aguda con la crónica no tratada. ${ }^{23}$

El ligamento cruzado anterior (LCA) sufre alteraciones en el número, diámetro y densidad de fibras de colágeno por el aumento de la tensión intrarticular. ${ }^{24}$

Petrigliano y $\mathrm{col}^{25}$ en condiciones de laboratorio, demostró que la sección del LCP y EPL resultan en un incremento significativo de la traslación posterior de la tibia durante el test de cajón posterior ( $10.5 \mathrm{~mm}$ en compartimiento medial y $16.5 \mathrm{~mm}$ en compartimiento lateral). Además de incrementarse significativamente la rotación externa al medir la traslación del compartimiento externo durante el dial test en $30^{\circ}(15.5 \mathrm{~mm})$ y $90^{\circ}(14.5 \mathrm{~mm})$. 
Parte de las razones por las que una lesión completa de la esquina posterolateral no se logra reparar con tratamiento conservador, está relacionada con la inestabilidad inherente del compartimiento FT externo, debido a que ambas superficies de contacto son convexas. Asociado a eso, la movilidad aumentada del menisco externo provee mucho menos efecto estabilizador ante incrementos excesivos en la movilidad articular que su contraparte medial más estable. En pacientes con genu varo asociado, esa inestabilidad inherente empeora a consecuencia de un momento aductor aumentado en el compartimiento medial, que genera tensión excesiva en la esquina posterolateral, la cual causa su elongación en el tiempo.

En la insuficiencia crónica posterolateral y genu varo concurrente, a menudo se presenta una inestabilidad funcional que conduce a un patrón de marcha característico en varus thrust, ${ }^{26,27}$ que incluye: aumento del momento aductor, rotación tibial externa y recurvatum. El resultado evolutivo final es un triple varo sintomático. Como lo describió Noyes y col, ${ }^{28}$ el término varo primario o geométrico se refiere al mal alineamiento óseo. El varo secundario se produce por separación del compartimiento FT externo debido a la insuficiencia de las partes blandas (elongación de las estructuras laterales). El triple varo es consecuencia de la insuficiencia de la esquina posterolateral y del pivote central producto de un daño más severo de las partes blandas (recurvatum y exceso de rotación tibial externa).

\section{Evaluación Clínica}

La historia de este tipo de pacientes puede aquejar síntomas inespecíficos de inestabilidad medio-lateral en grados próximos a la extensión completa de rodilla, que pueden describirse como dolor difuso o fallos en la deambulación, especialmente en terreno irregular y escaleras.

En el examen físico, la actitud en bipedestación puede evidenciar el signo de aprensión, con flexo de la rodilla afectada para evitar la incomodidad producida por el varo y recurvatum sintomático. La marcha en varus thrust (-Fig. 2) es el signo que evidencia la disfunción crónica de la esquina posterolateral.

Existen diversos test clínicos para valorar la $\mathrm{EPL}^{29}$ y $\mathrm{LCP}^{30}$ Existen pruebas estáticas y dinámicas, que presentan variación en su sensibilidad y especificidad, y que estarán determinadas, principalmente, por la experiencia de quien las realiza, por lo que la sumatoria de ellas permitirá una adecuada aproximación diagnóstica.

\section{Dentro de las Pruebas Para la Detección de Lesión del LCP Destacan}

El test de hundimiento posterior (posterior sag): en posición supina con ambas rodillas en flexión de $90^{\circ}$, se mantienen en posición los talones y se compara la traslación posterior pasiva anormal de la tibia afectada bajo una visión lateral. Se considera la prueba más sensible para lesión del LCP. ${ }^{30}$

El test de cajón posterior: en posición supina con las rodillas en $90^{\circ}$ de flexión y el examinador sentado en el pie del paciente para fijarlo. Se aplica una fuerza posterior

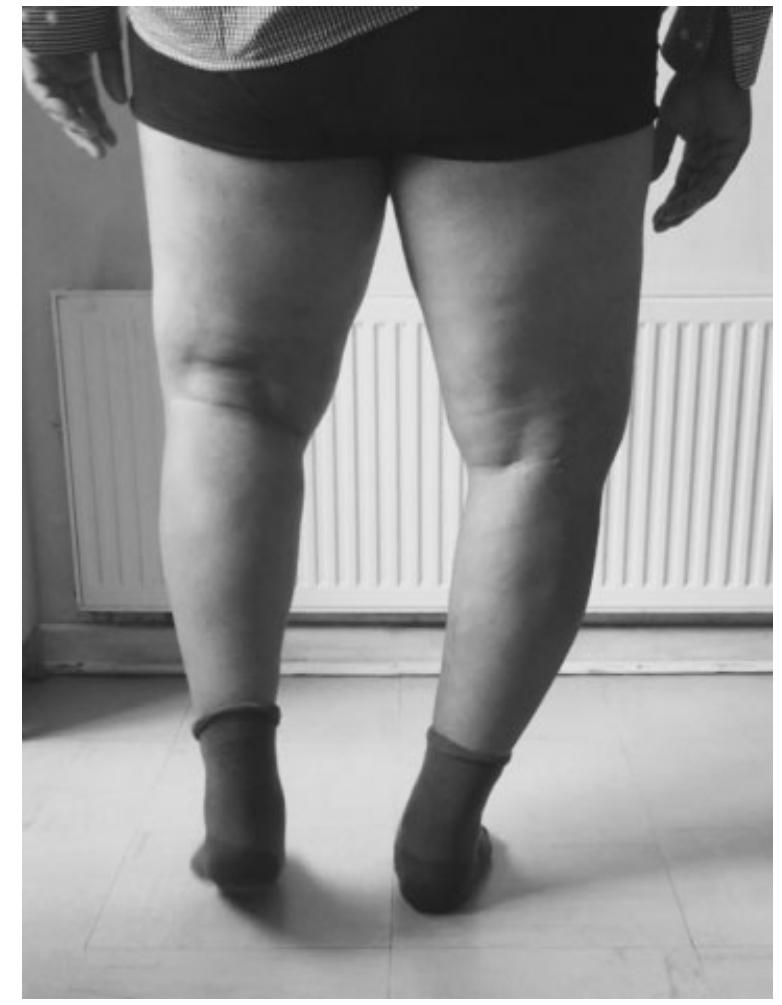

Fig. 2 Varus Thrust. Paciente con insuficiencia crónica de la esquina posterolateral (EPL) derecha. Se solicita realizar marcha habitual. Nótese la apertura excesiva del espacio articular femorotibial (FT) externo con aumento del varo en fase de apoyo de la extremidad inferior derecha.

sobre la diáfisis tibial anterior, comparando la traslación tibial posterior del lado afectado versus el lado sano.

El test de contracción activa cuadricipital: paciente en posición supina con la rodilla en flexión de $90^{\circ}$. Con el pie fijo, se solicita intentar deslizarlo distalmente, induciendo a una contracción cuadricipital. Si la tibia se traslada anteriormente más de $2 \mathrm{~mm}$ (desde una posición subluxada posterior), la prueba es positiva. Se considera el test más específico para la lesión del $\mathrm{LCP}^{30}$

El test del dial (Dial test): para ser realizado en posición supina, el paciente debe colocar sus extremidades inferiores en el extremo de la camilla para que un ayudante pueda estabilizar las rodillas en $30^{\circ}$ y $90^{\circ}$ de flexión. El examinador rota externamente la rodilla y compara la posición final de la tuberosidad anterior de la tibia lado a lado. En posición decúbito prono no es necesario un asistente. Se debe medir el ángulo muslo-pie en rotación externa de rodillas (-Fig. 3 ). El test es positivo si hay un aumento de al menos $10^{\circ}$ en la rotación tibial en comparación con el lado sano. En $30^{\circ}$ de flexión, un test positivo confirma la lesión aislada de la EPL. Luego se realiza la prueba en flexión de $90^{\circ}$. Si existe un incremento en la rotación externa, se asocia a una lesión combinada con el LCP.

El test de pivot shift reverso: paciente en posición supina con rodilla en flexión cercana a $90^{\circ}$ y pie en rotación externa. El examinador realiza un estrés en valgo sobre la rodilla mientras ella es lentamente extendida. Si existe 


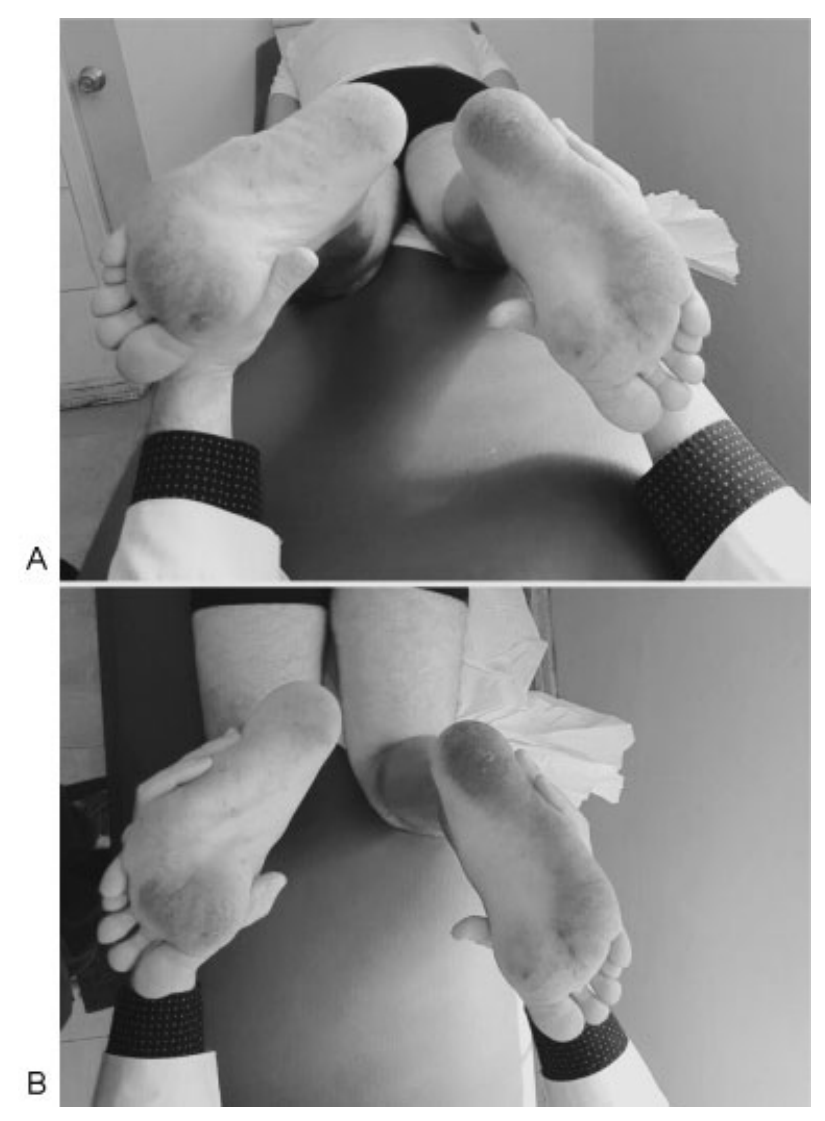

Fig. 3 Dial Test. Paciente con lesión crónica de la esquina posterolateral (EPL) izquierda. En A. nótese la diferencia $>10^{\circ}$ en el ángulo muslo-pie entre la rodilla afectada (izquierda) y sana cuando el examinador realiza el test con $30^{\circ}$ de flexión. En B., se realiza el test con $90^{\circ}$ de flexión. No hay mayor diferencia en la rotación externa entre ambas extremidades, por lo que se concluye que no existe lesión asociada del ligamento cruzado posterior (LCP).

lesión de la EPL, el platilllo tibial lateral pasa de un estado subluxado a uno abruptamente reducido en $25^{\circ}-30^{\circ}$ de flexión. Esa reducción es el resultado del cambio de función de la banda iliotibial, de flexor a extensor de rodilla.

El test de estrés en varo: la rodilla del paciente se estabiliza en extensión completa y luego en $20^{\circ}$ a $30^{\circ}$ de flexión aplicando una fuerza en varo. La cantidad de apertura del espacio femorotibial externo determina el grado de lesión (-Fig. 4). Un test positivo en extensión, determina la lesión combinada del LCL y LCA o LCP. En caso de apertura en $20^{\circ}-30^{\circ}$ de flexión y no en extensión, se correlaciona con la lesión aislada del LCL.

De forma clásica, según el sistema de evaluación de la rodilla del IKDC (International Knee Documentation Committee), las lesiones ligamentarias se pueden graduar en 4 niveles según la apertura articular (normal: 0-2mm, grado I: 3-5mm, grado II: 6-10 mm y grado III o lesión completa $>10 \mathrm{~mm}$ ). Laprade y $\mathrm{col}^{31}$ en un estudio cadavérico con radiografías tomadas en estrés seccionando de forma secuencial la esquina posterolateral y luego los ligamentos cruzados, evidenció que ese sistema sobrestima el grado de lesión. Descubrió que se puede inferir una lesión completa del LCL con 2,7mm de apertura lateral,

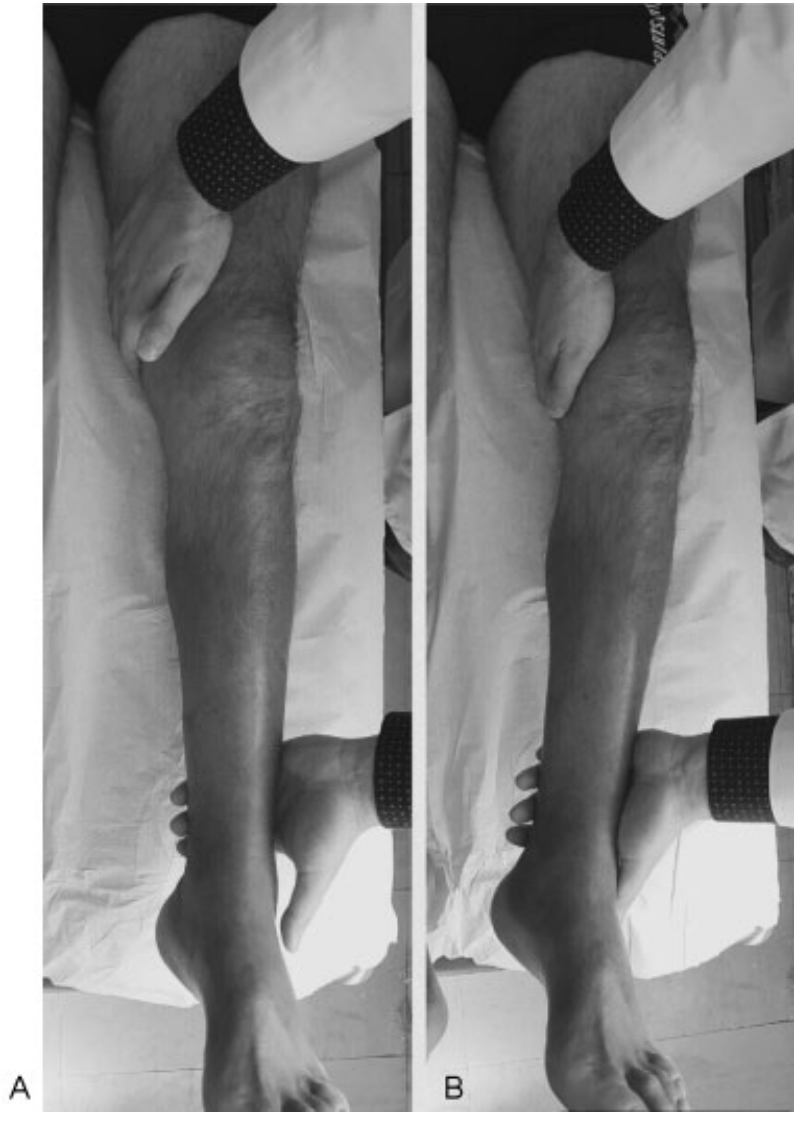

Fig. 4 Test de Estrés en Varo. Se prosigue con el examen del mismo paciente de figura previa. Se muestra examen físico de rodilla lesionada (izquierda). En A., se eleva extremidad inferior desde el talón para lograr la extensión completa de la rodilla, fijándola con la otra mano sobre el muslo del paciente. En B., se realiza la maniobra de estrés en varo con resultado positivo, evidenciando apertura del espacio femorotibial (FT) lateral. Se confirma así, una lesión combinada de la esquina posterolateral (EPL) y pivote central. En el caso particular, paciente presenta además un test de Lachmann y cajón anterior positivos, evidenciando una lesión asociada del ligamento cruzado anterior (LCA).

lesión completa de la EPL con $4 \mathrm{~mm}$, lesión combinada de EPLy LCA con $6,6 \mathrm{~mm}$ y lesión del LCA, LCP y EPL con 7,8mm en comparación con una rodilla intacta.

El test de recurvatum - rotación externa: paciente en posición supina y con ambas rodillas extendidas. El examinador eleva desde el hallux la extremidad inferior, estabilizando el muslo (-Fig. 5), resultando en hiperextensión y varo con rotación externa de la rodilla afectada. Su valor se expresa en grados negativos de movilidad articular o incremento del despegue del talón comparado con la extremidad contralateral. Por cada centímetro de diferencia en la altura del talón, existe un grado de recurvatum. ${ }^{10}$ Un test positivo se asocia a lesiones combinadas de EPL con LCA o LCP.

El test de cajón posterolateral: paciente en posición supina con rodilla en $90^{\circ}$ de flexión y pierna fija en $15^{\circ}$ rotación externa, mientras el examinador realiza una fuerza posterior con rotación externa sobre la tibia. Si existe discrepancia en el desplazamiento posterior y rotatorio respecto al lado sano, se considera lesión del LCL, tendón poplíteo y ligamento poplíteofibular. 


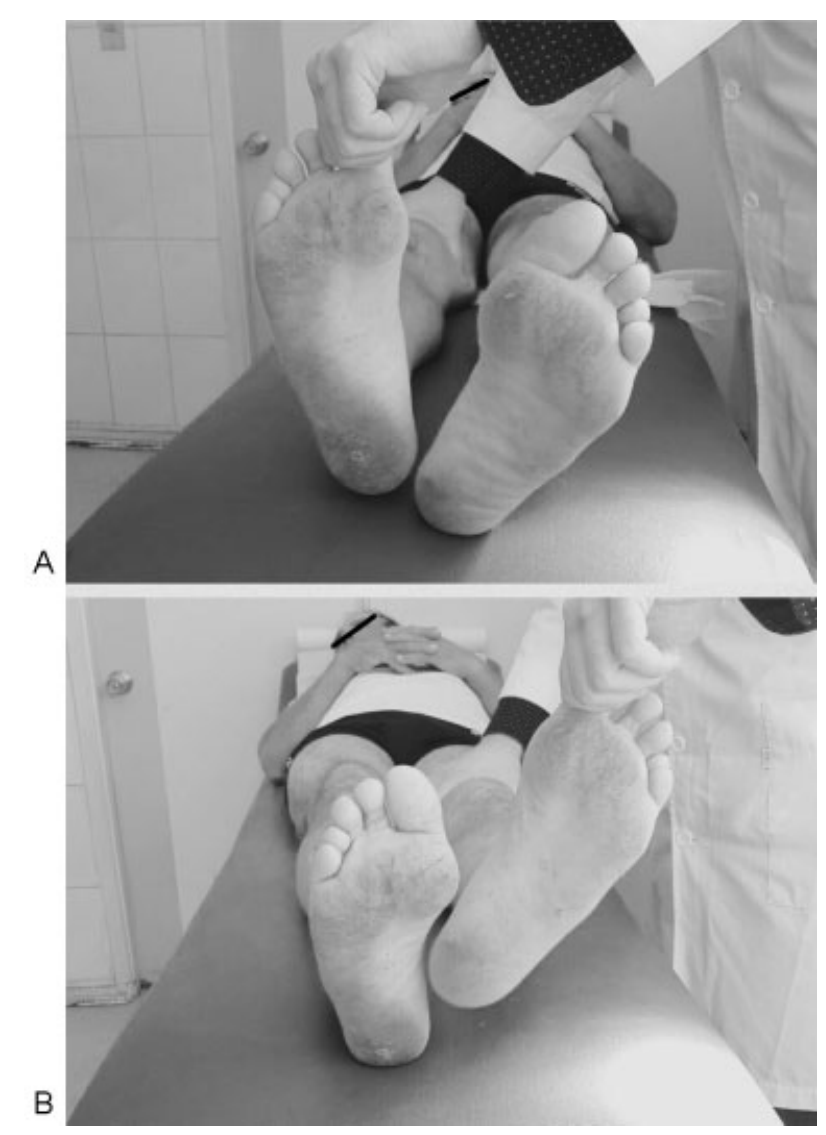

Fig. 5 Test Recurvatum - Rotación externa. Mismo paciente de figuras anteriores (-Figs. 3 y $\mathbf{4}$ ). En A., se realiza el test de la rodilla sana (derecha). Nótese que no hay despegue del talón desde la camilla cuando se eleva la extremidad inferior desde el hallux fijando el muslo. En B., test positivo. Nótese la acentuada elevación del talón, evidenciando recurvatum de rodilla izquierda asociado a la rotación externa excesiva en comparación con la rodilla contralateral bajo las mismas condiciones. Se confirma así, una lesión combinada de la esquina posterolateral (EPL) y pivote central (en el caso particular, del ligamento cruzado anterior).

\section{Nuestro Enfrentamiento}

En nuestro Hospital, es habitual observar la evolución natural de esta patología. Probablemente la oportunidad de la atención es un factor que influye en esa particularidad. La población desarrolla su actividad principalmente en el sector agrícola e industrial, en condiciones de alta exigencia articular, lo que genera detrimento acelerado de la función biomecánica ya evaluada en esta revisión. Al ser lesiones de cronicidad evidente, los pacientes generalmente no recuerdan un evento traumático específico. Sin embargo, es constante la presencia de un mal alineamiento en varo.

Consideramos a esas lesiones crónicas, transcurridos 3 meses de evolución ${ }^{27}$ desde el evento índice (cuando es posible consignarlo). Existen reportes que hablan de considerarlas crónicas tras 6 semanas de evolución. ${ }^{32}$ Ese límite es apenas mencionado en la literatura, sin embargo es de vital importancia establecerlo, ya que condiciona la elección del tipo de tratamiento.

En la anamnesis, se intenta descartar algún mecanismo traumático evidente (fuerzas aplicadas en el aspecto anteromedial de la tibia, hiperextensión y varo). Clínicamente se evalúa la aprensión en bipedestación, la marcha en busca de varus thrust, trastornos del alineamiento óseo subyacentes y los test específicos para valorar la EPL. Éstos deben realizarse siempre de forma bilateral, ya que por ejemplo, un test de pivot shift reverso puede resultar positivo en hasta el 35\% de las rodillas normales. ${ }^{33}$ Sólo el $28 \%$ de las lesiones de EPL ocurren de forma aislada, ${ }^{34}$ por lo que es de regla realizar un examen físico completo con especial énfasis en la evaluación del pivote central (LCA y LCP). Se requiere descartar lesiones meniscales, las que están presentes en el $36 \%$ de los casos crónicos. ${ }^{23}$

El estudio complementario incluye: radiografías de rodilla comparativas AP con carga, lateral y axial de rótulas. Telerradiografía de extremidades inferiores (como estudio imagenológico fundamental) y resonancia nuclear magnética de rodilla, con el fin de confirmar lesiones asociadas.

El manejo de la lesión crónica de la EPL se fundamenta en los hallazgos clínicos y radiológicos, basados en el entendimiento de la anatomía y biomecánica particular de cada paciente. De forma primaria, siempre consistirá en corregir el varus thrust mediante osteotomía tibial alta (según descripción a evaluar en la siguiente actualización sobre el tema, que trata sobre el razonamiento de esa elección quirúrgica).

\section{Discusión}

La evolución natural del genu varo o de una lesión del complejo posterolateral no diagnosticada, puede producir insuficiencia por elongación de las partes blandas, que conducen a la inestabilidad y al consecuentemente aumento del momento aductor durante la marcha. La EPL juega el rol más importante en resistirlo, siendo el LCL, LPF y TP las estructuras más destacadas. ${ }^{4} \mathrm{Su}$ disfunción crónica genera varus thrust, un potente factor de riesgo para la progresión de la artrosis FT medial. $^{35}$ Es de vital importancia el análisis clínico de la marcha para poder determinar esa característica dinámica.

En consecuencia, el examen físico detallado es la piedra angular del diagnóstico. Ninguno de los test específicos por si solo tiene elevada sensibilidad ni especificidad, por lo que es obligatorio la suma de ellos en la evaluación clínica.

\section{Conclusiones}

La inestabilidad crónica posterolateral de rodilla sigue siendo un tema desafiante desde la evaluación inicial hasta la resolución definitiva. La comprensión de la anatomía, biomecánica articular y las adaptaciones evolutivas de la rodilla para su complejo funcionamiento, son esenciales para el razonamiento diagnóstico y terapéutico de esas lesiones.

\section{Agradecimientos}

A los Doctores: Carlos Bolomey E., Javier Cayazaya D. y Pedro Gómez C. del Hospital Clínico FUSAT de Rancagua, por sus valiosos aportes y enseñanzas aplicadas en esta revisión. A todo el equipo de Traumatología del Hospital Ricardo Valenzuela Sáez de Rengo, por el apoyo mostrado en la realización de este artículo; Doctores Julio Hernández E., 
Joel Hernández DL., Alexander Pérez A., Francisco Neumann C., Mario Humeres R. y Patricia Salazar M.

\section{Conflicto de Intereses}

La realización de este estudio no cuenta con conflicto de intereses por parte del autor.

\section{Declaración y Verificación de la Presentación}

Este trabajo no ha sido previamente publicado ni presentado simultáneamente en otra revista y tiene la autorización del autor y su establecimiento asistencial.

\section{Bibliografía}

1 Fanelli GC, Edson CJ. Posterior cruciate ligament injuries in trauma patients: Part II. Arthroscopy 1995;11(05):526-529

2 James EW, LaPrade CM, LaPrade RF. Anatomy and biomechanics of the lateral side of the knee and surgical implications. Sports Med Arthrosc Rev 2015;23(01):2-9

3 Gollehon DL, Torzilli PA, Warren RF. The role of the posterolateral and cruciate ligaments in the stability of the human knee. A biomechanical study. J Bone Joint Surg Am 1987;69(02):233-242

4 LaPrade RF, Ly TV, Wentorf FA, Engebretsen L. The posterolateral attachments of the knee: a qualitative and quantitative morphologic analysis of the fibular collateral ligament, popliteus tendon, popliteofibular ligament, and lateral gastrocnemius tendon. Am J Sports Med 2003;31(06):854-860

5 Moulton SG, Fontboté C, Cram T, LaPrade RF. Posterolateral reconstruction of the knee: Surgical technique with 2 grafts. Oper Tech Sports Med 2015;23:331-337

6 LaPrade RF, Bollom TS, Wentorf FA, Wills NJ, Meister K. Mechanical properties of the posterolateral structures of the knee. Am J Sports Med 2005;33(09):1386-1391

7 LaPrade RF, Tso A, Wentorf FA. Force measurements on the fibular collateral ligament, popliteofibular ligament, and popliteus tendon to applied loads. Am J Sports Med 2004;32(07):1695-1701

8 McCarthy M, Camarda L, Wijdicks CA, Johansen S, Engebretsen L, Laprade RF. Anatomic posterolateral knee reconstructions require a popliteofibular ligament reconstruction through a tibial tunnel. Am J Sports Med 2010;38(08):1674-1681

9 LaPrade RF, Morgan PM, Wentorf FA, Johansen S, Engebretsen L. The anatomy of the posterior aspect of the knee. An anatomic study. J Bone Joint Surg Am 2007;89(04):758-764

10 Morgan PM, LaPrade RF, Wentorf FA, Cook JW, Bianco A. The role of the oblique popliteal ligament and other structures in preventing knee hyperextension. Am J Sports Med 2010;38(03):550-557

11 LaPrade RF, Terry GC. Injuries to the posterolateral aspect of the knee. Association of anatomic injury patterns with clinical instability. Am J Sports Med 1997;25(04):433-438

12 LaPrade CM, Civitarese DM, Rasmussen MT, LaPrade RF. Emerging Updates on the Posterior Cruciate Ligament: A Review of the Current Literature. Am J Sports Med 2015;43(12):3077-3092

13 Harner CD, Janaushek MA, Kanamori A, Yagi M, Vogrin TM, Woo SL. Biomechanical analysis of a double-bundle posterior cruciate ligament reconstruction. Am J Sports Med 2000;28(02):144-151

14 Kennedy NI, Wijdicks CA, Goldsmith MT, et al. Kinematic analysis of the posterior cruciate ligament, part 1: the individual and collective function of the anterolateral and posteromedial bundles. Am J Sports Med 2013;41(12):2828-2838

15 Amis AA. Biomechanics of high tibial osteotomy. Knee Surg Sports Traumatol Arthrosc 2013;21(01):197-205

16 Shelburne KB, Torry MR, Pandy MG. Muscle, ligament, and jointcontact forces at the knee during walking. Med Sci Sports Exerc 2005;37(11):1948-1956
17 Foroughi N, Smith R, Vanwanseele B. The association of external knee adduction moment with biomechanical variables in osteoarthritis: a systematic review. Knee 2009;16(05):303-309

18 Teichtahl AJ, Davies-Tuck ML, Wluka AE, Jones G, Cicuttini FM. Change in knee angle influences the rate of medial tibial cartilage volume loss in knee osteoarthritis. Osteoarthritis Cartilage 2009;17(01):8-11

19 Bellemans J, Colyn W, Vandenneucker H, Victor J. The Chitranjan Ranawat award: is neutral mechanical alignment normal for all patients? The concept of constitutional varus. Clin Orthop Relat Res 2012;470(01):45-53

20 Vandekerckhove PT, Matlovich N, Teeter MG, MacDonald SJ, Howard JL, Lanting BA. The relationship between constitutional alignment and varus osteoarthritis of the knee. Knee Surg Sports Traumatol Arthrosc 2016

21 Miller MD, Cooper DE, Fanelli GC, Harner CD, LaPrade RF. Posterior cruciate ligament: current concepts. Instr Course Lect 2002;51:347-351

22 Ramaniraka NA, Terrier A, Theumann N, Siegrist O. Effects of the posterior cruciate ligament reconstruction on the biomechanics of the knee joint: a finite element analysis. Clin Biomech (Bristol, Avon) 2005;20(04):434-442

23 Geissler WB, Whipple TL. Intraarticular abnormalities in association with posterior cruciate ligament injuries. Am J Sports Med 1993;21(06):846-849

24 Ochi M, Murao T, Sumen Y, Kobayashi K, Adachi N. Isolated posterior cruciate ligament insufficiency induces morphological changes of anterior cruciate ligament collagen fibrils. Arthroscopy 1999;15(03):292-296

25 Petrigliano FA, Suero EM, Voos JE, Pearle AD, Allen AA. The effect of proximal tibial slope on dynamic stability testing of the posterior cruciate ligament- and posterolateral corner-deficient knee. Am J Sports Med 2012;40(06):1322-1328

26 Badhe NP, Forster IW. High tibial osteotomy in knee instability: the rationale of treatment and early results. Knee Surg Sports Traumatol Arthrosc 2002;10(01):38-43

27 Arthur A, LaPrade RF, Agel J. Proximal tibial opening wedge osteotomy as the initial treatment for chronic posterolateral corner deficiency in the varus knee: a prospective clinical study. Am J Sports Med 2007;35(11):1844-1850

28 Noyes FR, Barber-Westin SD, Hewett TE. High tibial osteotomy and ligament reconstruction for varus angulated anterior cruciate ligament-deficient knees. Am J Sports Med 2000;28(03):282-296

29 Cooper JM, McAndrews PT, LaPrade RF. Posterolateral corner injuries of the knee: anatomy, diagnosis, and treatment. Sports Med Arthrosc Rev 2006;14(04):213-220

30 Kopkow C, Freiberg A, Kirschner S, Seidler A, Schmitt J. Physical examination tests for the diagnosis of posterior cruciate ligament rupture: a systematic review. J Orthop Sports Phys Ther 2013;43(11):804-813

31 LaPrade RF, Heikes C, Bakker AJ, Jakobsen RB. The reproducibility and repeatability of varus stress radiographs in the assessment of isolated fibular collateral ligament and grade-III posterolateral knee injuries. An in vitro biomechanical study. J Bone Joint Surg Am 2008;90(10):2069-2076

32 Chahla J, Moatshe G, Dean CS, LaPrade RF. Posterolateral Corner of the Knee: Current Concepts. Arch Bone Jt Surg 2016;4(02):97-103

33 Cooper DE. Tests for posterolateral instability of the knee in normal subjects. Results of examination under anesthesia. J Bone Joint Surg Am 1991;73(01):30-36

34 Geeslin AG, LaPrade RF. Location of bone bruises and other osseous injuries associated with acute grade III isolated and combined posterolateral knee injuries. Am J Sports Med 2010;38 (12):2502-2508

35 Chang A, Hayes K, Dunlop D, et al. Thrust during ambulation and the progression of knee osteoarthritis. Arthritis Rheum 2004; 50(12):3897-3903 